\title{
Nonlinear Adaptive Control of an Aerial Manipulation System
}

\author{
Emre Yilmaz ${ }^{1}$, Hammad Zaki ${ }^{1}$, Mustafa Unel ${ }^{1,2}$
}

\begin{abstract}
This paper presents the nonlinear adaptive control of a quadrotor endowed with a 2 degrees of freedom (DOF) manipulator. By considering the quadrotor and the robot arm as a combined system, complete modeling of the aerial manipulation system (AMS) has been presented using the Euler-Lagrange method. A hierarchical nonlinear control scheme which consists of outer and inner control loops has been utilized. Model Reference Adaptive Controller (MRAC) is designed for the outer loop where the required command signals are generated to force the quadrotor to move on a reference trajectory in the presence of uncertainties and reaction forces coming from the manipulator. For the inner loop, the attitude dynamics of the quadrotor and the dynamics of the 2-DOF robotic arm are considered as a fully actuated 5-DOF unified part of the AMS. Nonlinear adaptive control has been utilized for the low-level controller where the changes in inertias and the masses have been tackled along with the reaction forces acting on the attitude part of the AMS. The proposed technique has been validated through simulations in two different scenarios.
\end{abstract}

\section{INTRODUCTION}

In recent years, unmanned aerial vehicles (UAVs) have greatly attracted the research community towards aerial robotics. Among UAVs, the quadrotor is one of the most used kinds due to its vertical takeoff and landing (VTOL) capability. Previously, quadrotors were utilized for passive tasks such as surveillance, inspection, remote sensing, and so on. Recently, quadrotors have been used for active purposes where grasping and manipulation operations are done through aerial manipulation system. However, the complete switching from "passive" into "active" tasks requires a mechanical arm in order to perform more complex actions. Equipping a quadrotor with a manipulator system makes the control more challenging due to its unstable nonlinear dynamics and the added coupling effects.

Nowadays, the functionality of a UAV is extended by equipping it with a manipulator which is used to deliver loads [1] and [2]. Other than the load carrying, UAVs equipped with a robotic arm could be used to perform different tool operations at the places where it is difficult for humans to reach. In that case, a UAV has to impose force with fixed tools on the external environment [3] and [4]. Tool operations and gripping an object result in a change in the center of gravity, hence resulting in instability due to coupling effects. Therefore, there is a need to compensate for the coupling effects and the change of mass along with the

\footnotetext{
*This work was not supported by any organization

${ }^{1}$ Faculty of Engineering and Natural Sciences, Sabanci University, Tuzla, Istanbul 34956, Turkey

${ }^{2}$ Integrated Manufacturing Technologies Research and Application Center, Sabanci University, Istanbul, Turkey

\{emreyilm, hammadzaki, munel\}@sabanciuniv.edu
}

change of inertias and reaction forces. A PID controller was developed for a quadrotor equipped with a gripper where the center of mass offset had been taken into account and linear least-squares methods had been utilized to estimate payload parameters and payload inertia in the controller design in [5]. Computed torque method has been applied in [6] to reduce the effects of the manipulator and also $\mathcal{L}_{1}$ adaptive control had been proposed to compensate for the unknown payload and inertial effects. Heredia et. al. presented an integral backstepping controller for an octaquad with 7 DOF manipulator [7]. In order to achieve stability with a high payload, an admittance controller for the manipulator arm had been implemented, which was a position-based Cartesian impedance controller. The admittance controller commanded the desired Cartesian position for the end effector tool center point. Cano et. al. in [8] presented a variable parameter integral backstepping (VPIB) control for AMS where stabilization of attitude controller had been focused to deal with a varying center of mass and inertia. Kim et. al. [9] used 2-DOF manipulator with quadrotor where the whole dynamics of the quadrotor and manipulator system is considered as a unified system and an adaptive sliding mode technique is employed for the control. Model reference adaptive control based on Lyapunov stability analysis had been proposed in [11]. In continuation of the previous work Orsag et. al. presented a hybrid adaptive control scheme for the aerial manipulation system (AMS) where gain scheduling and Lyapunov based adaptive control had been utilized. Passivity based controller had been presented by Lee et. al. in [13] where unknown parameters of a payload are estimated by an online estimator based on the parametrization of the aerial manipulator dynamics. Adaptive backstepping and terminal sliding mode controller were utilized in [14] and the presented scheme is tested for both motionless and wavering conditions of the robotic arm connected to the quadrotor. Nonlinear Model Predictive Control had been presented for 3-D trajectory tracking control of a quadrotor with the 3DOF serial manipulator in [15].

Different vision-based techniques had been also presented to control the complex dynamics of an AMS. In [16] an image based visual servoing (IBVS) controller for dynamic vision-based grasping and perching for an aerial manipulation system had been developed. The geometric visual controller had been developed that considered the second order dynamics (in contrast to most visual servoing controllers that assume first order dynamics). Kim et. al. presented vision guided aerial manipulation system with a stereo camera on an end effector of a robotic arm where the vision guidance approach using an image-based visual servoing 
(IBVS) had been presented [17]. Based on the combined model, a passivity-based adaptive controller for both position and velocity controls had been designed. Integrated visionbased guiding system for aerial manipulation system had been presented in [18] where visual processing scheme had been used for object detection and manipulator positioning. The stereo camera had been employed for efficient aligning of the UAV according to the position of the end effector.

In this work, a quadrotor equipped with a 2-DOF manipulator has been taken into account. The Euler-Lagrange method has been utilized to model the aerial manipulation system (AMS). A nonlinear hierarchical control structure has been used for the the control of both positional and attitude dynamics of the quadrotor disturbed by the reaction forces coming from the manipulator part. Whole dynamics of the AMS is divided into two parts: The first part consists of the positional dynamics and the second part combines attitude dynamics of the quadrotor with the 2-DOF manipulator dynamics, making it a 5-DOF fully actuated system. Model Reference Adaptive Control (MRAC) has been utilized as an upper level controller which is responsible for generating required virtual controls to enable the quadrotor to follow the desired trajectory. The nonlinear adaptive control algorithm [19] has been employed as a low level controller to tackle the change in the mass and the inertia of the AMS. Full nonlinear dynamics of the attitude of the quadrotor and manipulator have been taken into account while designing the nonlinear adaptive controller. For simulations, two separate scenarios have been considered. In the first scenario, a circular trajectory has been followed by the quadrotor when the joint angles of the manipulator are fixed. In the second scenario, the quadrotor hovers at a certain altitude where the end-effector of the manipulator draws a circle. Simulation results show the effectiveness of the proposed controller in both cases.

The paper is organized as follows: Section II describes the kinematic and dynamics of the aerial manipulation system. Section III elaborates the controller design part. Results are presented in section IV. Section V contains a summary along with future work.

\section{SySTEM MODEL}

Kinematics and dynamics of the aerial manipulator system (AMS) consists of the quadrotor combined with the 2 DOF robotic arm are presented in this section. The AMS dynamics are derived regarding Lagrange formalization [9]- [10].

\section{A. Kinematics for the Aerial Manipulation System}

Coordinate frames of the combined system is defined in Fig. 1. $\mathrm{O}_{W}, \mathrm{O}_{B}, \mathrm{O}_{1}$ and $\mathrm{O}_{2}$ are namely the inertial frame, the body frame of the quadrotor and link 1 and link 2 respectively. Regarding notations, $R_{b}^{w}$ defines a transformation from $O_{B}$ to $O_{W}$. Similarly $R_{i}^{b}$ stands for a transformation from $O_{i}$ to $O_{B}$. The subscript $\mathrm{i}=1,2$ denotes the link number. The generalized coordinate variables are defined as

$$
q=\left[\begin{array}{lll}
p & \Phi & \zeta
\end{array}\right]^{T}
$$

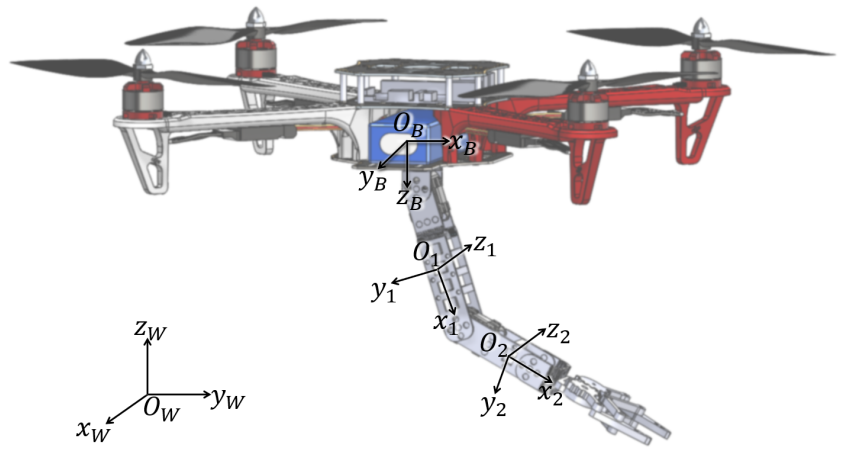

Fig. 1: Coordinate frames of the system

where $p=[X, Y, Z]$ denotes the position of the quadrotor in inertial frame, $\Phi=[\phi, \theta, \psi]$ represents the attitudes of the quadrotor and $\zeta=\left[\zeta_{1}, \zeta_{2}\right]$ defines the manipulator joint angles.

The translational and angular velocities in the inertial frame for the quadrotor are denoted as $\dot{p}$ and $\omega$.

$$
\begin{gathered}
\dot{p}=R_{b}^{w} \dot{p}^{b} \\
\omega=R_{b}^{w} \omega^{b}=T \dot{\Phi}
\end{gathered}
$$

where $\omega^{b}$ is the angular velocities in body frame of the quadrotor and $T$ is the transformation matrix from Euler rates $(\dot{\Phi})$ to angular velocities $(\omega)$.

The position of the center of mass $p_{i}$ of each link $i$ in the inertial frame are related as

$$
p_{i}=p+R_{b}^{w} p_{i}^{b}
$$

where $p_{i}^{b}$ is the position of center of mass in body frame of each link $i$.

The translational and angular velocities in the inertial frame for each link $i$ are denoted as $\dot{p}_{i}$ and $\omega_{i}$.

$$
\begin{gathered}
\dot{p}_{i}=\dot{p}+\dot{R}_{b}^{w} p_{i}^{b}+R_{b}^{w} \dot{p}_{i}^{b} \\
\omega_{i}=\omega+R_{b}^{w} J_{t} \dot{\zeta}
\end{gathered}
$$

where $\dot{p}_{i}^{b}=J_{t} \dot{\zeta}$, and $\dot{R}_{b}^{w}=S\left(\omega_{b}\right) R_{b}^{w}$ and $\omega_{i}^{b}=J_{r} \dot{\zeta}$. $J_{t}$ and $J_{r}$ are the Jacobian matrices of the translations and rotations of links respectively. $S(w)$ defines the skewsymmetric matrix.

Above relations are converted into matrix forms as following

$$
\begin{gathered}
\dot{p}=\left[\begin{array}{lll}
I_{3 \times 3} & 0_{3 \times 3} & 0_{3 \times 2}
\end{array}\right] \dot{q} \triangleq \mathcal{T}_{1} \dot{q} \\
\omega=\left[\begin{array}{lll}
0_{3 \times 3} & T & 0_{3 \times 2}
\end{array}\right] \dot{q} \triangleq \mathcal{T}_{2} \dot{q} \\
\dot{p}_{i}=\left[\begin{array}{lll}
I_{3 \times 3}-S\left(R_{b}^{w} p_{i}^{b}\right) T & R_{b}^{w} J_{t, i}
\end{array}\right] \dot{q} \triangleq \mathcal{T}_{3} \dot{q} \\
\omega_{i}=\left[\begin{array}{lll}
0_{3 \times 3} T & R_{b}^{w} J_{r, i}
\end{array}\right] \dot{q} \triangleq \mathcal{T}_{4} \dot{q}
\end{gathered}
$$

\section{B. Dynamics for the Aerial Manipulation System}

Dynamics of the aerial manipulation system is derived by the Euler-Lagrange formulation. The total kinetic energy is computed as

$$
\mathcal{K}=\mathcal{K}_{b}+\sum_{i=1}^{2} \mathcal{K}_{i}
$$

where

$$
\mathcal{K}_{b}=\frac{1}{2} \dot{p}^{T} m_{b} \dot{p}+\frac{1}{2} \omega^{T}\left(R_{b}^{w}\right) I_{b}\left(R_{b}^{w}\right)^{T} \omega
$$


and

$$
\mathcal{K}_{i}=\frac{1}{2} \dot{p}^{T} m_{i} \dot{p}+\frac{1}{2} \omega_{i}^{T}\left(R_{b}^{w} R_{i}^{b}\right) I_{i}\left(R_{b}^{w} R_{i}^{b}\right)^{T} \omega_{i}
$$

The total potential energy is computed as

$$
\mathcal{U}=m_{b} g e_{3}^{T} p+\sum_{i=1}^{2} m_{i} g e_{3}^{T}\left(p+R_{b}^{w} p_{i}^{b}\right)
$$

The dynamic equation of the aerial manipulation system can be written in a compact form as

$$
M(q) \ddot{q}+C(q, \dot{q}) \dot{q}+G(q)=\tau
$$

The total kinetic energy can be rewritten in terms of the inertia matrix $M(q)$ as

$$
\mathcal{K}=\frac{1}{2} \dot{q}^{T} M(q) \dot{q}
$$

In light of equations (7)-(10), $M(q)$ can be computed as

$$
\begin{gathered}
M(q)=\mathcal{T}_{1}^{T} m_{b} \mathcal{T}_{1}+\mathcal{T}_{2}^{T}\left(R_{b}^{w}\right) I_{b}\left(R_{b}^{w}\right)^{T} \mathcal{T}_{2}+ \\
\sum_{i=1}^{2} \mathcal{T}_{3}^{T} m_{i} \mathcal{T}_{3}+\mathcal{T}_{4}^{T}\left(R_{b}^{w} R_{i}^{b}\right) I_{i}\left(R_{b}^{w} R_{i}^{b}\right)^{T} \mathcal{T}_{4}
\end{gathered}
$$

The elements of the Coriolis matrix are calculated as follows:

$$
c_{k j}=\sum_{i=1}^{8} \frac{1}{2}\left\{\frac{\partial m_{k j}}{\partial q_{i}}+\frac{\partial m_{k i}}{\partial q_{j}}-\frac{\partial m_{i j}}{\partial q_{k}}\right\} \dot{q}_{i}
$$

$G(q)$ is calculated as

$$
G(q)=\frac{\partial \mathcal{U}}{\partial q}
$$

Inputs for the quadrotor $\left(U_{1}, U_{2}, U_{3}, U_{4}\right)$ and manipulator actuators $\left(\tau_{1}, \tau_{2}\right)$ are calculated as

$$
\left[\begin{array}{c}
U_{1} \\
U_{2} \\
U_{3} \\
U_{4} \\
\tau_{1} \\
\tau_{2}
\end{array}\right]=\left[\begin{array}{ccc}
R_{b}^{w}(3,3) & 0 & 0 \\
0 & \left(\left(R_{b}^{w}\right)^{T} T\right)^{-1} & 0 \\
0 & 0 & I_{2 \times 2}
\end{array}\right]^{-1}\left[\begin{array}{c}
\tau(3) \\
\vdots \\
\tau(8)
\end{array}\right]
$$

where $U_{1}$ is the total thrust, $U_{2}, U_{3}$ and $U_{4}$ are rolling, pitching, and yawing moments of the quadrotor respectively. $\tau_{1}$ and $\tau_{2}$ represent the joint torques of the manipulator.

\section{Controller Design}

A hierarchical control structure is a useful technique where separate controllers for the positional and attitude dynamics of the quadrotor are designed as an upper level and low level controllers respectively. For the upper level controller, Model Reference Adaptive Controller (MRAC) is used to generate the required command signals to control the Cartesian position of the quadrotor in the presence of uncertainties and reaction forces acting on the positional dynamics. These command signals are then converted to the desired attitude angles for the lower level attitude controller. A nonlinear adaptive control is employed as a low level controller where the fully actuated 5-DOF nonlinear dynamics of the AMS including attitude dynamics of the UAV along with the manipulator. Since nonlinear adaptive control is used, therefore linearization of the system is not required to compensate the uncertainties and the change of inertias due to the coupling effect of the manipulator. A block diagram is presented in Fig. 2 to describe the overall closed loop control structure.

\section{A. Model Reference Adaptive Control Design}

Model Reference Adaptive Control is used as an upper level controller for the positional dynamics of the quadrotor. This controller generates the necessary command signals for the calculation of the total thrust and the desired attitude angles. Those are used by the low level nonlinear controller, in the $\mathrm{X}, \mathrm{Y}$ and $\mathrm{Z}$ directions, to make the AMS follow its desired trajectory. Uncertainty in mass and reaction forces due to the coupling effect of the manipulator are handled by the online modification of the control parameters based on the trajectory error.

1) Reference Model Design: Consider the following positional dynamics of a UAV [20]- [21]

$$
\underline{\dot{X}}=F \underline{X}+G_{n} \Delta(u+D), \quad y=H \underline{X}
$$

where $\underline{X}=[X, Y, Z, \dot{X}, \dot{Y}, \dot{Z}], y$ is the plant output,

$$
\begin{gathered}
F=\left[\begin{array}{ll}
0_{3 \times 3} & I_{3 \times 3} \\
0_{3 \times 3} & 0_{3 \times 3}
\end{array}\right], \quad G_{n}=\left[\begin{array}{c}
0_{3 \times 3} \\
I_{3 \times 3}
\end{array}\right] \frac{1}{m_{n}}, \\
D=\left[\begin{array}{c}
0_{2 \times 1} \\
m g
\end{array}\right], H=\left[\begin{array}{ll}
I_{3 \times 3} & 0_{3 \times 3}
\end{array}\right], \Delta=m_{n} / m
\end{gathered}
$$

where $m$ and $m_{n}$ are the actual and nominal mass of the vehicle respectively. Consider the following control law,

$$
u_{n}=K_{x}^{T} \underline{X}+K_{r}^{T} r-D
$$

The nominal closed loop dynamics is obtained as

$$
\underline{\dot{X}}_{n}=\left(F+G_{n} K_{x}^{T}\right) \underline{X}_{n}+G_{n} K_{r}^{T} r
$$

where $K_{x}$ can be calculated by pole placement of LQR. Defining $F_{m}=F+G_{n} K_{x}^{T}$, the nominal plant output is obtained as

$$
y_{n}=H\left(s I-F_{m}\right)^{-1} G_{n} K_{r}^{T} r
$$

For constant $r$, the steady state plant output can be calculated as

$$
y_{s s}=-H F_{m}^{-1} G_{n} K_{r}^{T} r
$$

using $K_{r}^{T}=-\left(H F_{m}^{-1} G_{n}\right)^{-1}$, it is obtained as

$$
\lim _{t \rightarrow \infty}\left(y_{n}-r\right)=0
$$

As a result, the reference model dynamics is determined as

$$
\underline{\dot{X}}_{m}=F_{m} \underline{X}_{m}+G_{m} r
$$

where

$$
F_{m}=F+G_{n} K_{x}^{T}, \quad G_{m}=G_{n} K_{r}^{T}=-G_{n}\left(H F_{m}^{-1} G_{n}\right)^{-1}
$$




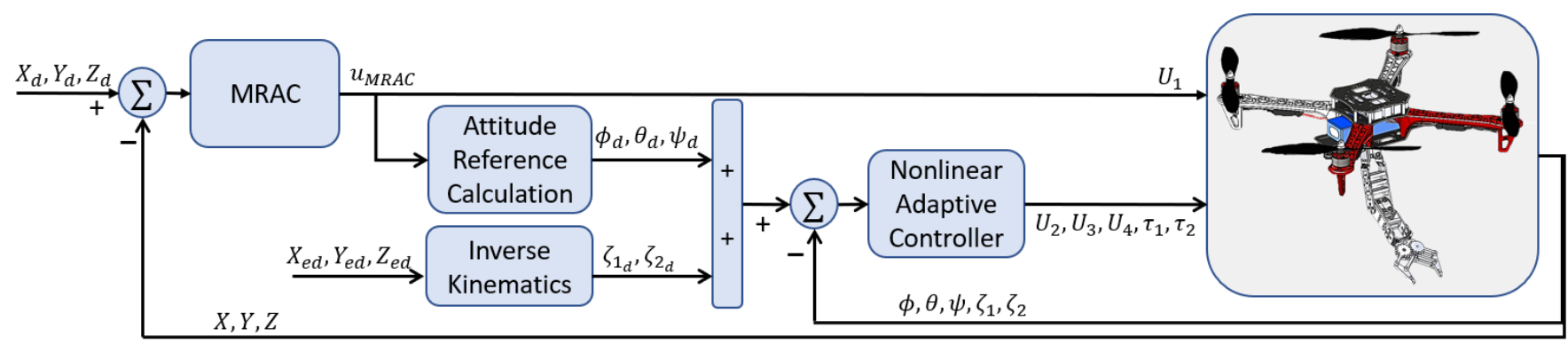

Fig. 2: Overall Control System Architecture

2) Adaptive Control: Consider the following adaptive controller [21]:

$$
u_{M R A C}=\hat{K}_{x}^{T} \underline{X}+\hat{K}_{r}^{T} r+\hat{D}
$$

with the adaptive laws

$$
\begin{gathered}
\dot{\hat{K}}_{x}=-\Gamma_{x}\left(\underline{X} e^{T} P G_{n}-\sigma_{x}\|e\| \hat{K}_{x}\right) \\
\dot{\hat{K}}_{r}=-\Gamma_{r}\left(r e^{T} P G_{n}-\sigma_{r}\|e\| \hat{K}_{r}\right) \\
\dot{\hat{D}}^{T}=-\Gamma_{d}\left(e^{T} P G_{n}-\sigma_{D}\|e\| \hat{D}\right)
\end{gathered}
$$

where $e=\underline{X}-\underline{X}_{m}, \Gamma_{x}, \Gamma_{r}, \Gamma_{d}$ are the adaptive gain, $\sigma_{x}$, $\sigma_{r}$ and $\sigma_{D}$ are positive scaler gains and $P$ is the symmetric solution of the Lyapunov equation

$$
F_{m}^{T} P+P F_{m}=-Q
$$

where $Q$ is a positive definite matrix.

3) Attitude Reference Calculation: From the equation (28), using the first two components of the $u_{M R A C}$ and assuming small roll and pitch angles, one can derive the desired attitude angles of the quadrotor as

$$
\left[\begin{array}{l}
\theta_{d} \\
\phi_{d}
\end{array}\right]=\frac{1}{U_{1}}\left[\begin{array}{cc}
\cos \psi^{*} & \sin \psi^{*} \\
\sin \psi^{*} & -\cos \psi^{*}
\end{array}\right]\left[\begin{array}{l}
u_{M R A C}^{1} \\
u_{M R A C}^{2}
\end{array}\right]
$$

where $U_{1}=\left\|u_{M R A C}\right\|, \psi_{d}=\psi^{*}$ is the desired fixed yaw angle and $u_{M R A C}^{i}$ is the $i^{\text {th }}$ component of the $u_{M R A C}, i=$ $1,2,3$.

\section{B. Nonlinear Adaptive Control Design}

The nonlinear adaptive controller [19] is employed to force the AMS to follow its desired attitude angles, in the presence of uncertainties and change of inertias due to manipulator. Consider the attitude and manipulator dynamics as

$$
M\left(\alpha_{\omega}\right) \dot{\Omega}_{\omega}+C\left(\alpha_{\omega}, \Omega_{\omega}\right) \Omega_{\omega}=u^{\prime}
$$

where $\alpha_{w}=\left[\phi, \theta, \psi, \zeta_{1}, \zeta_{2}\right]^{T}$. Through a long but tedious procedure, one can parameterize this equation linearly in terms of moments of inertia of the UAV and the manipulator; i.e.

$$
Y\left(\alpha_{\omega}, \dot{\alpha}_{\omega}, \ddot{\alpha}_{\omega}\right) I_{A M S}=u^{\prime}
$$

where $I_{A M S}=\left[I_{x x}, I_{y y}, I_{z z}, I_{1}, I_{2}\right]$. In order to get rid of the acceleration term $\ddot{\alpha}_{\omega}$ which is difficult to obtain, the following filtered error is defined;

$$
s=\dot{\tilde{\alpha}}_{\omega}+\Lambda_{s} \tilde{\alpha}_{\omega}
$$

where $\tilde{\alpha}_{\omega}=\alpha_{\omega}-\alpha_{\omega d}, \alpha_{\omega d}$ is the desired value of $\alpha_{\omega}$ and $\Lambda_{s}$ is a symmetric positive definite matrix. Equation (36) can be rewritten as

$$
s=\dot{\alpha}_{\omega}-\dot{\alpha}_{\omega r}
$$

where $\dot{\alpha}_{\omega r}=\dot{\alpha}_{\omega d}-\Lambda_{s} \tilde{\alpha}_{\omega}$

A matrix $Y^{\prime}=Y^{\prime}\left(\alpha_{\omega}, \dot{\alpha}_{\omega}, \dot{\alpha}_{\omega, r}, \ddot{\alpha}_{\omega, r}\right)$ can be defined, to be used in linear parameterization, as in the case of equation (35) such that

$$
M\left(\alpha_{\omega}\right) \dot{\Omega}_{\omega}+C\left(\alpha_{\omega}, \Omega_{\omega}\right)=Y^{\prime}\left(\alpha_{\omega}, \dot{\alpha}_{\omega}, \dot{\alpha}_{\omega, r}, \ddot{\alpha}_{\omega, r}\right) I_{A M S}
$$

It can be shown that the following nonlinear controller

$$
u_{A M S}=Y^{\prime} \hat{I}_{A M S}-K_{D} s
$$

where $K_{D}$ is positive definite matrix and $\hat{I}$ is an estimate of the uncertain parameter $I$, with an adaptive law

$$
\dot{\hat{I}}_{A M S}=-\Gamma_{I} Y^{\prime T} s
$$

where $\Gamma_{I}$ is the adaption rate, stabilizes the closed loop system and makes the error $\tilde{\alpha}_{\omega}$ converge to zero.

\section{RESUltS AND DisCUSSION}

Simulation results for two different scenarios are presented in this section, where the proposed controller is implemented using the nonlinear dynamics of aerial manipulation system. The reference model for the design of the model reference adaptive controller is determined using a linear quadratic regulator (LQR). It is assumed that UAV mass is uncertain with a $20 \%$ uncertainty. In the simulations, external disturbances acting on the system are generated through Dryden Wind model and measurement noise is also taken into account. Simulation parameters are tabulated in Table I.

TABLE I: Simulation Parameters

\begin{tabular}{lll}
\hline Symbol & Description & Magnitude \\
\hline$m_{b}$ & Mass of the UAV & $2.8 \mathrm{~kg}$ \\
$m_{1}$ & Mass of the link 1 & $0.1 \mathrm{~kg}$ \\
$m_{2}$ & Mass of the link 2 & $0.1 \mathrm{~kg}$ \\
$L_{1}$ & Length of the link 1 & $0.15 \mathrm{~m}$ \\
$L_{2}$ & Length of the link 2 & $0.15 \mathrm{~m}$ \\
$I_{x x}$ & Moment of inertia of the UAV ( $x_{B}$ axis) & $12 \mathrm{e}-3 \mathrm{kgm}^{2}$ \\
$I_{y y}$ & Moment of inertia of the UAV $\left(y_{B}\right.$ axis $)$ & $12 \mathrm{e}-3 \mathrm{kgm}^{2}$ \\
$I_{z z}$ & Moment of inertia of the UAV $\left(z_{B}\right.$ axis $)$ & $16 \mathrm{e}-3 \mathrm{kgm}^{2}$ \\
$I_{1}$ & Moment of inertia of the link 1 & $1.875 \mathrm{e}-4 \mathrm{kgm}^{2}$ \\
$I_{2}$ & Moment of inertia of the link 2 & $1.875 \mathrm{e}-4 \mathrm{kgm}^{2}$ \\
\hline
\end{tabular}




\section{A. First Scenario}

In the first scenario, joint angles of the manipulator of AMS are fixed and the quadrotor draws a circle in $X Z$ plane after reaching a certain altitude.

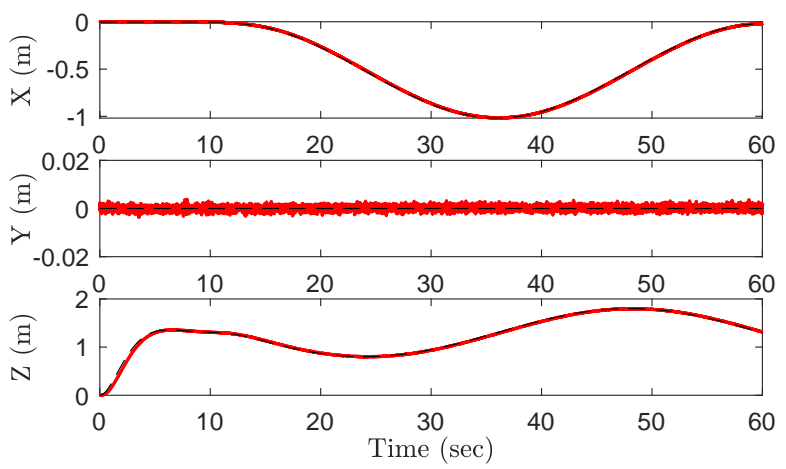

Fig. 3: Cartesian positions of the quadrotor vs Time (Desired=dashed, Actual=red solid)

Figure 3 shows the cartesian position plots of the quadrotor, which follows the desired trajectory smoothly under external disturbances varying from $-0.5 \mathrm{~N}$ to $0.5 \mathrm{~N}$. Based on the tracking errors provided in Table II, the position tracking of the quadrotor is quite accurate with RMSE in the range of $0.001 \mathrm{~m}-0.024 \mathrm{~m}$. In Table II, $e_{X e}, e_{Y e}$, and $e_{Z e}$ denote the positional errors for the end-effector of the manipulator. Note that there is no significant difference between tracking performances of the quadrotor and the manipulator endeffector. Worst-case position errors are also very small, i.e around $0.11 \mathrm{~m}$, for both the quadrotor and the manipulator.

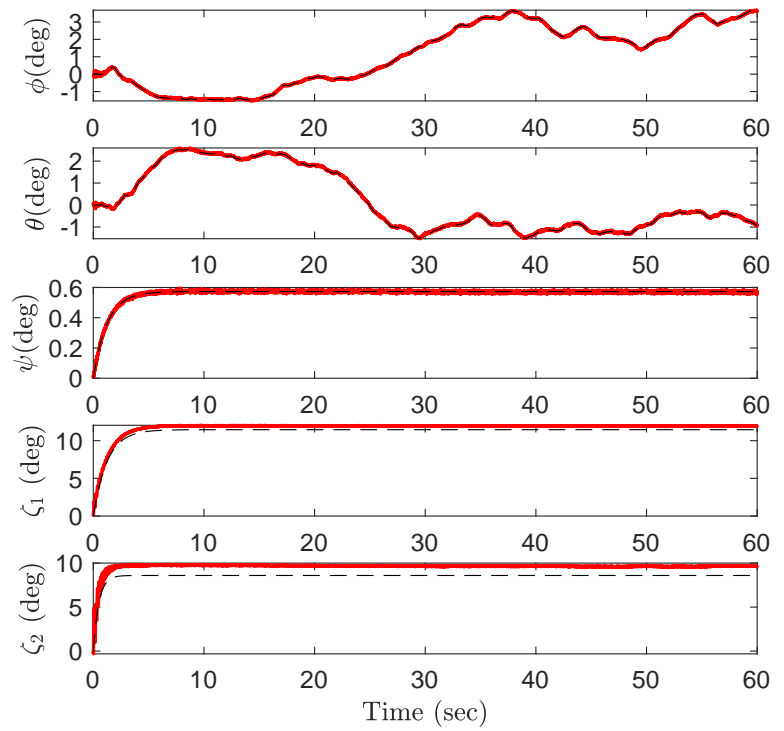

Fig. 4: Euler angles and manipulator joint angles vs Time (Desired=dashed, Actual=red solid)

Attitude angles of the AMS have been depicted in Figure 4 where both the quadrotor and the manipulator follow their desired trajectories smoothly under external disturbances varying from $-0.8 \mathrm{Nm}$ to $0.8 \mathrm{Nm}$. According to the Table II, the attitude tracking of the quadrotor is very accurate with RMSE ranging from $0.024^{\circ}$ to $0.059^{\circ}$. For the manipulator end-effector, RMSE values are in the range of $0.5^{\circ}-1.091^{\circ}$.
Inner loop nonlinear adaptive controller is quite robust in achieving the desired performance. Worst-case attitude errors are $0.57^{\circ}$ and $3.53^{\circ}$ for the quadrotor and the manipulator, respectively.

TABLE II: Tracking errors of the AMS for scenario I

\begin{tabular}{llllll}
\hline Criterion & $\begin{array}{l}\text { RMS } \\
\text { Errors }\end{array}$ & $\begin{array}{l}\text { Max } \\
\text { Errors }\end{array}$ & Criterion & $\begin{array}{l}\text { RMS } \\
\text { Errors }\end{array}$ & $\begin{array}{l}\text { Max } \\
\text { Errors }\end{array}$ \\
\hline$e_{X}(m)$ & 0.005 & 0.012 & $e_{\phi}(\mathrm{deg})$ & 0.028 & 0.223 \\
$e_{Y}(m)$ & 0.001 & 0.004 & $e_{\theta}(\mathrm{deg})$ & 0.024 & 0.160 \\
$e_{Z}(m)$ & 0.024 & 0.110 & $e_{\psi}(\mathrm{deg})$ & 0.059 & 0.576 \\
$e_{X e}(m)$ & 0.005 & 0.012 & $e_{\zeta_{1}}(\mathrm{deg})$ & 0.500 & 1.271 \\
$e_{Y e}(m)$ & 0.001 & 0.004 & $e_{\zeta_{2}}(\mathrm{deg})$ & 1.091 & 3.533 \\
$e_{Z e}(m)$ & 0.026 & 0.115 & & & \\
\hline
\end{tabular}

\section{B. Second Scenario}

In the second scenario, the quadrotor is enabled to hover at a certain altitude and the manipulator is allowed to draw a circle in $X Z$ plane.

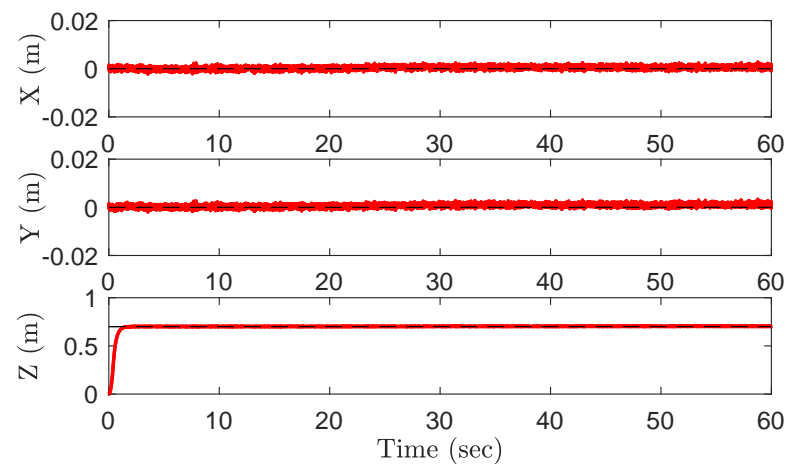

Fig. 5: Cartesian positions of the quadrotor vs Time (Desired=dashed, Actual=red solid)

Cartesian position plots for this scenario have been shown in Figure 5, where the quadrotor shows a stable hovering at the desired altitude under external disturbances varying from $-1 \mathrm{~N}$ to $2 \mathrm{~N}$. According to the tracking errors given in Table III, the position tracking error has RMSE values in the range of $0.001 \mathrm{~m}$ to $0.053 \mathrm{~m}$. As before, the difference between tracking performances of the quadrotor and the manipulator end-effector is not significant. Worst-case position errors are around $0.7 \mathrm{~m}$ for both the quadrotor and the manipulator.

Attitude angles of the quadrotor and manipulator joint angles have been depicted in Figure 6 where it can be noticed that the system follows the desired angles satisfactorily under external disturbances varying from $-4 \mathrm{Nm}$ to $4 \mathrm{Nm}$. The attitude tracking of the quadrotor is very accurate and precise with RMSE in the range of $0.002^{\circ}-0.098^{\circ}$. For the manipulator end-effector, RMSE values are in the range of $0.085^{\circ}-0.431^{\circ}$. Worst-case attitude errors are $0.59^{\circ}$ and $2.01^{\circ}$ for the quadrotor and the manipulator, respectively. It is clear that the inner loop controller successfully rejects disturbances acting on the system.

The performance of the developed controllers can be improved by fine tuning of the controller parameters, which require more control efforts. 

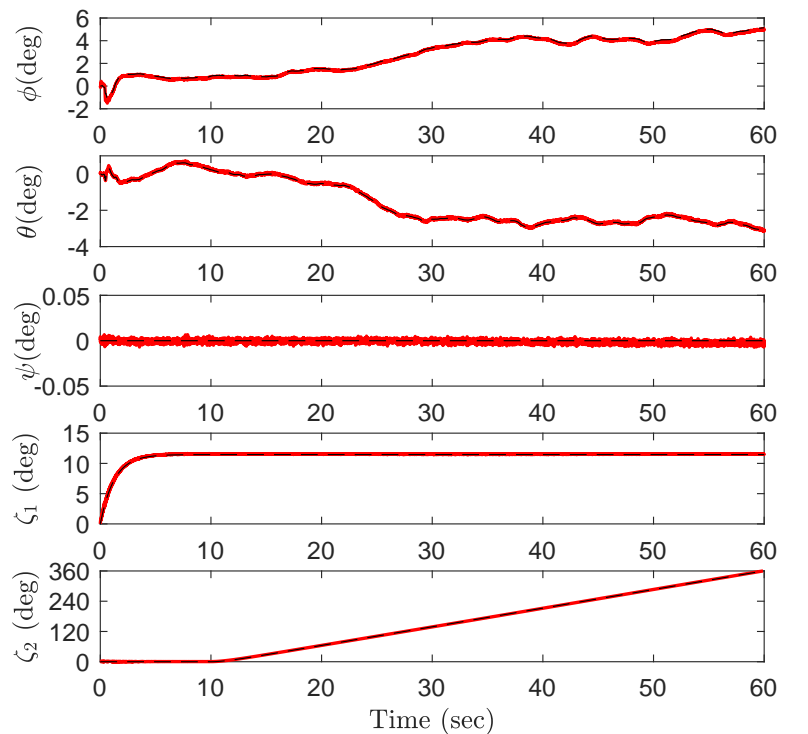

Fig. 6: Euler angles and manipulator joint angles vs Time (Desired=dashed, Actual=red solid)

TABLE III: Tracking errors of the AMS for scenario II

\begin{tabular}{llllll}
\hline Criterion & $\begin{array}{l}\text { RMS } \\
\text { Errors }\end{array}$ & $\begin{array}{l}\text { Max } \\
\text { Errors }\end{array}$ & Criterion & $\begin{array}{l}\text { RMS } \\
\text { Errors }\end{array}$ & $\begin{array}{l}\text { Max } \\
\text { Errors }\end{array}$ \\
\hline$e_{X}(m)$ & 0.001 & 0.003 & $e_{\phi}($ deg $)$ & 0.098 & 0.598 \\
$e_{Y}(m)$ & 0.001 & 0.003 & $e_{\theta}($ deg $)$ & 0.036 & 0.129 \\
$e_{Z}(m)$ & 0.053 & 0.700 & $e_{\psi}($ deg $)$ & 0.002 & 0.007 \\
$e_{X e}(m)$ & 0.002 & 0.005 & $e_{\zeta_{1}}($ deg $)$ & 0.085 & 0.524 \\
$e_{Y e}(m)$ & 0.001 & 0.004 & $e_{\zeta_{2}}($ deg $)$ & 0.431 & 2.016 \\
$e_{Z e}(m)$ & 0.054 & 0.706 & & & \\
\hline
\end{tabular}

\section{CONCLUSION AND FUTURE WORK}

In this work, nonlinear adaptive control of an aerial manipulation system (AMS) consisting of a quadrotor with a 2-DOF manipulator is tackled. In order to control the positional dynamics of the AMS in the presence of mass uncertainty and reaction forces, model reference adaptive control (MRAC) has been utilized. Using the command signals generated by the MRAC and making small angle approximations, desired attitude angles are calculated analytically for the low level controller. Attitude dynamics of the quadrotor and 2-DOF manipulator dynamics are combined as a 5-DOF fully actuated system. Nonlinear adaptive control is implemented for this 5-DOF rotational dynamics where uncertainties in mass and inertia are considered. Performance of the proposed method is validated through simulations where two cases have been taken into account. A circle drawing task with the end-effector of the manipulator is set to be a goal for the AMS. During the first case, the quadrotor is allowed to draw a circle by fixing the manipulator angles while during the second case the manipulator is allowed to draw a circle by forcing the quadrotor to hover at a certain altitude. Simulation results revealed that the proposed method provides satisfactory performance despite the uncertainties in mass, inertias and reaction forces.

As a future work, the performance of the proposed method will be tested on a real experimental platform.

\section{REFERENCES}

[1] P. Cruz, and R. Fierro, "Autonomous lift of a cable-suspended load by an unmanned aerial robot", In Control Applications (CCA), 2014 IEEE Conference, Oct. 2014, pp. 802-807.

[2] A. Faust, I. Palunko, P. Cruz, R. Fierro, and L. Tapia, "Automated aerial suspended cargo delivery through reinforcement learning", Artificial Intelligence, 2017, 381-398.

[3] G. Gioioso, M. Ryll, D. Prattichizzo, H.H. Bulthoff, and A. Franchi, "Turning a near-hovering controlled quadrotor into a 3D force effector", In Robotics and Automation (ICRA), 2014 IEEE International Conference, May 2014, pp. 6278-6284.

[4] H. W. Wopereis, J. J. Hoekstra, T. H. Post, G. A. Folkertsma, S. Stramigioli, and M. Fumagalli, "Application of substantial and sustained force to vertical surfaces using a quadrotor", In 2017 IEEE International Conference on Robotics and Automation (ICRA), May 2017, pp. 2704-2709.

[5] D. Mellinger, Q. Lindsey, M. Shomi, and V. Kumar, "Design, modeling, estimation and control for aerial grasping and manipulation", In Intelligent Robots and Systems (IROS), 2011 IEEE/RSJ International Conference, Sep. 2011, pp. 2668-2673.

[6] R. M. Jones, D. Sun, Barsi, G. Haberfeld, A. Lakshmanan, T. Marinho, and N. Hovakimyan, "Design and control of a small aerial manipulator for indoor environments", In AIAA Information Systems-AIAA Infotech@ Aerospace, 2017,pp. 1374.

[7] G. Heredia, A. E. Jimenez-Cano, I. Sanchez, D. Lorente, V. Vega, J. Braga, and A. Ollero, "Control of a multirotor outdoor aerial manipulator", In Intelligent Robots and Systems (IROS 2014), 2014 IEEE/RSJ International Conference, Sep. 2014, pp. 3417-3422.

[8] A. E. Jimenez-Cano, J. Martin, G. Heredia, A. Ollero, and R. Cano, "Control of an aerial robot with multi-link arm for assembly tasks", In 2013 IEEE International Conference on Robotics and Automation, May 2013, pp. 4916-4921.

[9] S. Kim, S. Choi, and H. J. Kim, "Aerial manipulation using a quadrotor with a two dof robotic arm", In Intelligent Robots and Systems (IROS), 2013 IEEE/RSJ International Conference, Nov. 2013, pp. 4990-4995.

[10] M. W. Spong, S. Hutchinson, and M. Vidyasagar, Robot modeling and control, New York: Wiley, vol. 3, 2006, pp. 187-227.

[11] M. Orsag, C. Korpela, S. Bogdan, and P. Oh, "Lyapunov based model reference adaptive control for aerial manipulation", In Unmanned Aircraft Systems (ICUAS), 2013 International Conference on, May 2013, pp. 966-973.

[12] M. Orsag, C. M. Korpela, S. Bogdan, and P. Y. Oh, "Hybrid adaptive control for aerial manipulation", Journal of intelligent \& robotic systems, vol. 73, no. 1-4, Sep. 2014, pp. 693-707.

[13] H. Lee, S. Kim, and H. J. Kim, "Control of an aerial manipulator using on-line parameter estimator for an unknown payload", In 2015 IEEE International Conference on Automation Science and Engineering (CASE), August 2015.

[14] R. Jiao, W. Chou, R. Ding, and M. Dong, "Adaptive robust control of quadrotor with a 2-degree-of-freedom robotic arm", Advances in Mechanical Engineering, vol. 8, 2018.

[15] D. Lunni, A. Santamaria-Navarro, R. Rossi, P. Rocco, L. Bascetta, and J. Andrade-Cetto, "Nonlinear model predictive control for aerial manipulation", In Unmanned Aircraft Systems (ICUAS), 2017 International Conference on, June 2017.

[16] J. Thomas, G. Loianno, K. Sreenath, and V. Kumar, "Toward image based visual servoing for aerial grasping and perching", In Robotics and Automation (ICRA), 2014 IEEE International Conference on, May 2014.

[17] S. Kim, H. Seo, S. Choi, and H. J. Kim, "Vision-guided aerial manipulation using a multirotor with a robotic arm", IEEE/ASME Transactions On Mechatronics, vol. 4, 2016, pp. 1912-1923.

[18] C. Kanellakis, M. Terreran, D. Kominiak, and G. Nikolakopoulos, "On vision enabled aerial manipulation for multirotors", In Emerging Technologies and Factory Automation (ETFA), 2017 22nd IEEE International Conference on, Sep. 2017, pp. 1-7.

[19] J.-J. E. Slotine, and W. Li, Applied nonlinear control. Engle Wood Cliffs, NJ: Prentice-Hall, 1991

[20] E. Lavretsky, "Adaptive control: Introduction, overview, and applications", In Lecture notes from IEEE Robust and Adaptive Control Workshop, May 2008.

[21] Y. Yildiz, M. Unel, and A. E. Demirel, "Nonlinear hierarchical control of a quad tilt-wing UAV: An adaptive control approach", International Journal of Adaptive Control and Signal Processing, vol. 9, 2017, pp. $1245-1264$. 